\title{
Bio-inspired strategy for controlled dopamine polymerization in basic solutions $\uparrow$
}

\author{
Xin Du, ${ }^{\mathrm{a}, \mathrm{b}}$ Linxian Li, ${ }^{\mathrm{b}}$ Farid Behboodi Sadabad, ${ }^{\mathrm{b}}$ Alexander Welle, ${ }^{\mathrm{c}, \mathrm{d}}$ Junsheng Li, \\ Stefan Heissler, ${ }^{\mathrm{C}}$ Huijie Zhang, ${ }^{\mathrm{e}}$ Nicolas Plumeré ${ }^{\mathrm{e}}$ and Pavel A. Levkin* ${ }^{\mathrm{b}, \mathrm{f}}$
}

\begin{abstract}
Polydopamine (PD) coatings, inspired by the adhesive behavior of the mussel foot, have attracted a lot of attention because of the simplicity, generalizability, and the capability for their secondary modification. However, many possible applications of PD coatings cannot be realized due to the difficulty in controlling dopamine polymerization under basic conditions. Here, we report a new method for the light-triggered control of both the onset and termination of dopamine polymerization under basic conditions. We demonstrate that dopamine polymerization in basic solutions can be efficiently inhibited by adding small quantities of sodium ascorbate (vitamin C) that reduces reactive dopamine quinone and delays dopamine polymerization. UV irradiation of this solution, however, leads to instantaneous dopamine polymerization, thereby making light-driven spatial and temporal control of dopamine polymerization under basic conditions possible. This method is inspired by the antioxidant role of vitamin $\mathrm{C}$ in the human body. The mechanism of the light-driven SA-controlled dopamine polymerization has been studied using spectroscopic and electroanalytical methods. By this new method we can control dopamine polymerization without changing the commonly used conditions for the formation of PD coatings, making this method compatible with all existing applications for PD coatings.
\end{abstract}

\section{Introduction}

Since the first report in $2007,{ }^{1}$ polydopamine (PD) coatings, inspired by the adhesive behavior of the mussel foot, have attracted a lot of attention. ${ }^{2-10}$ Dopamine (DA) rapidly polymerizes in basic solutions (normally in buffers at $\mathrm{pH} 8.5$ ), resulting in a thin PD layer formed on the immersed surface. This PD coating is reactive and can be further functionalized by thiols, amines or metal ions. ${ }^{1}$ The simplicity, generalizability, and the capability for secondary modification of PD coatings offer an ideal solution for surface functionalization in a

${ }^{a}$ School of Biological Sciences \& Medical Engineering, Southeast University, Nanjing, China

${ }^{b}$ Institute of Toxicology and Genetics, Karlsruhe Institute of Technology,

76021 Karlsruhe, Germany. E mail: pavel.levkin@kit.edu

${ }^{c}$ Institute of Functional Interfaces, Karlsruhe Institute of Technology,

76021 Karlsruhe, Germany

${ }^{d}$ Karlsruhe Nano Micro Facility, Karlsruhe Institute of Technology, 76021 Karlsruhe, Germany

${ }^{e}$ Institute of Organic Chemistry, Karlsruhe Institute of Technology, 76021 Karlsruhe, Germany

${ }^{f}$ Center for Electrochemical Sciences Molecular Nanostructures,

Ruhr Universität Bochum, Universitätsstrasse 150, 44780 Bochum, Germany

$\dagger$ Electronic supplementary information (ESI) available: Experimental details and supplementary figures. See DOI: 10.1039/c7py00051k variety of different applications from various fields ranging from drug delivery to 3D object surface functionalization. ${ }^{11-25}$

One drawback of the DA polymerization method is the lack of control of the progression of the reaction under basic conditions. DA spontaneously polymerizes as soon as a basic DA solution is formed, neither the starting point nor the termination of the polymerization can be controlled in this case. This can cause problems (contamination of devices, poor reproducibility, non-uniformity of the coating) when DA polymerization has to be combined with some common technologies, such as dip-pen nanolithography (DPN), microfluidic systems, inkjet printing, 3D or micro-contact printing $(\mu \mathrm{CP})$ etc., thereby significantly limiting possible applications of PD coatings. Photolithography and PD patterning under basic conditions are also challenging due to spontaneous polymerization. Some strategies have been developed to control dopamine polymerization by inhibiting polymerization in acidic or neutral $\mathrm{pH}^{26-28}$ However, to achieve such an inhibition effect, the solution has to be kept at low $\mathrm{pH}$ and the composition of the dopamine solutions are far from those normally used.

On the other hand, base-triggered DA polymerization has many advantages, for example, the method is very simple; ${ }^{1}$ normally the kinetics of DA polymerization and deposition in basic solutions are much faster than those in neutral or acidic solutions; ${ }^{26,29}$ and using oxygen as the oxidant can avoid possible impurities and contamination of the resulting PD 
film. ${ }^{30-32}$ Thus, most of the reported applications use base triggered polymerization of DA..$^{2,3,8,33}$ Considering the two aspects above, a controllable DA polymerization in basic solutions is highly desired.

It is interesting that the biosynthesis of eumelanin, having a similar structure to polydopamine ${ }^{9}$ and functioning as a pigment in the human body, ${ }^{34}$ is reminiscent to the synthesis of PD. The process starts from the oxidation of tyrosine or L-3,4-dihydroxyphenylalanine (L-DOPA) in the presence of oxygen and tyrosinase as a catalyst, ${ }^{34}$ followed by the oligomerization and polymerization of dopaquinones into eumelanin (Fig. S1a†). People with melanin overproduction (skin pigment disorder) are often treated with vitamin $\mathrm{C}$ (ascorbic acid, or its sodium salt state, sodium ascorbate), since it can reduce the quinones formed during the biosynthesis of eumelanin to catechols and thus inhibit eumelanin formation..$^{35,36}$

Another important effect of vitamin $\mathrm{C}$ is the protection from sunlight damage through the reaction with reactive oxygen species (ROS) formed under sunlight irradiation (Fig. S1b $\dagger$ ). ${ }^{36,37}$ Inspired by these two natural processes, we propose a method for controlling both the onset and termination of DA polymerization under basic conditions. We demonstrate here that DA polymerization in basic solutions can be efficiently inhibited by adding a very small amount of sodium ascorbate (SA), while a short pulse of UV irradiation leads to the rapid consumption of SA and reinitiation of DA polymerization (Fig. 1).

A mechanistic study including spectroscopic and electroanalytical methods revealed that SA reduces quinones produced in situ, into catechols thereby inhibiting DA polymerization. UV light, however, significantly accelerates the rates of both the oxidative consumption of SA as well as oxidation of DA, thereby triggering the DA polymerization. Thus, by com-

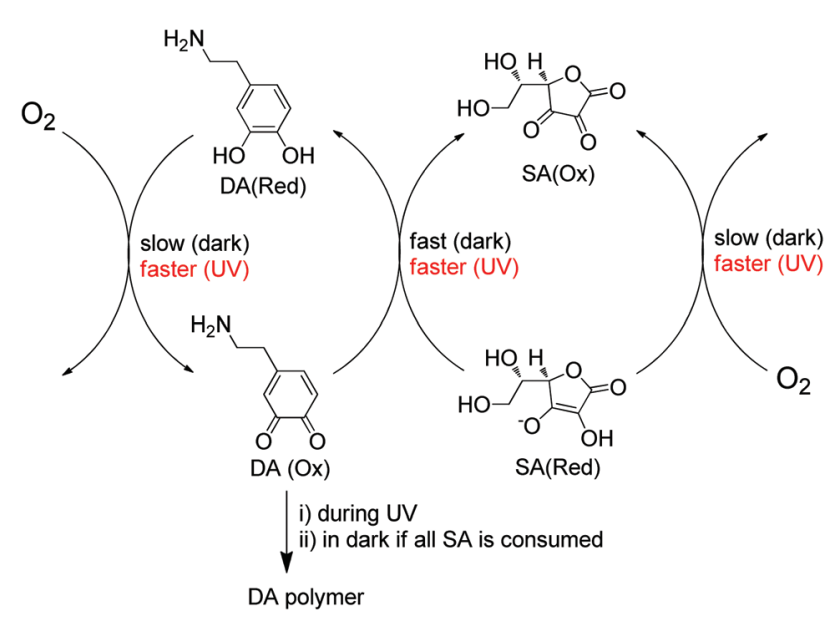

Fig. 1 Schematic illustration of the principle of controlling dopamine (DA) polymerization in basic solutions with the combination of UV and sodium ascorbate (SA). In the dark, the reducing agent (SA) present in the solution maintains DA in its reduced state, thus inhibiting DA polymerization. Exposing the solution to UV light leads to increased oxi dation rates of both DA and SA, resulting in the subsequent onset of DA oxidation and polymerization. bining SA and UV-irradiation, a controllable DA polymerization in basic solution could be demonstrated for the first time.

\section{Results and discussion}

DA polymerization is induced by an initial oxidation step. ${ }^{1}$ With base catalysis, DA is oxidized by oxygen to form DA quinone, followed by the intramolecular Michael addition, further oxidation, rearrangement and polymerization to form the final PD. ${ }^{38-40}$ The whole process is similar to the biosynthesis of eumelanin, therefore we hypothesized that DA polymerization under basic conditions could also be inhibited by the addition of SA as a reducing reagent. In order to investigate this hypothesis, the kinetics of DA polymerization in basic solutions at $\mathrm{pH} 8.5$ in the presence of SA was studied. DA solutions $\left(2 \mathrm{mg} \mathrm{mL}^{-1}\right)$ with SA concentrations from $0 \mathrm{wt} \%$ to $1 \mathrm{wt} \%$ (with respect to the DA, corresponding to the concentration of SA from 0 to $0.02 \mathrm{mg} \mathrm{mL}^{-1}$, respectively) were prepared and their time-dependent absorption at $420 \mathrm{~nm}$ was recorded (Fig. 2a). Fig. S2 $\uparrow$ shows the corresponding UV-vis absorption curves of the DA solutions at different time points. The results show that the kinetics of DA polymerization can be significantly decelerated even with a very small amount of SA added (Fig. 2a). With the addition of $0.1 \mathrm{wt} \%-1 \mathrm{wt} \%$ of SA (with respect to $2 \mathrm{mg} \mathrm{mL} \mathrm{m}^{-1} \mathrm{DA}$, i.e. SA concentration 0.002-0.02 $\mathrm{mg} \mathrm{mL}^{-1}$ ), DA polymerization was completely inhibited for 20-180 min. By addition of $5 \mathrm{wt} \%$ SA (with respect to $\mathrm{DA}$, i.e. $0.1 \mathrm{mg} \mathrm{mL}^{-1}$ ), $\mathrm{DA}$ polymerization can be completely stopped for $24 \mathrm{~h}$.

In the dark experiment, we proved that, similar to its role in melanogenesis, SA is an efficient inhibitor for DA polymerization. On the other hand, in the human body SA also works
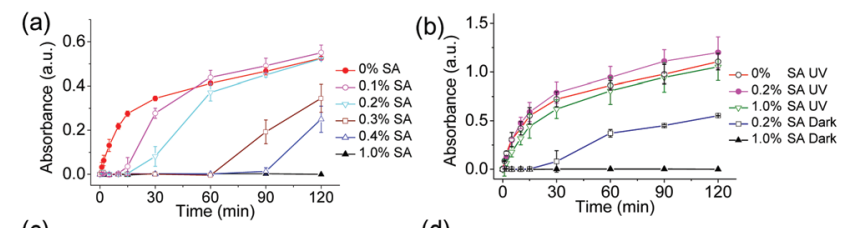

(c)

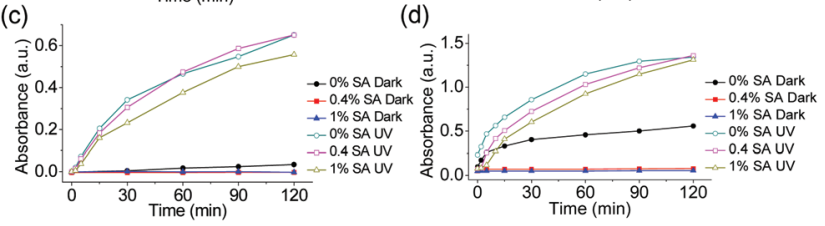

Fig. 2 Effect of SA on DA polymerization with and without UV irradiation. The graphs show absorbance of DA solutions at $420 \mathrm{~nm}$. DA concentration in all experiments was $2 \mathrm{mg} \mathrm{mL}{ }^{1}$. (a) DA polymerization (at $\mathrm{pH} 8.5$, Tris buffer $10 \mathrm{mM}$ ) in the dark in the presence of SA with different concentrations: 0 wt $\%, 0.1$ wt $\%, 0.2$ wt $\%, 0.3$ wt $\%, 0.4$ wt $\%$ and 1 wt\% with respect to DA. (b) DA polymerization (at $\mathrm{pH} 8.5$ ) both under UV light and in the dark in the presence of SA of different concen trations. Compare with DA polymerization in the dark $(0.2 \mathrm{wt} \%$ and $1 \mathrm{wt} \% \mathrm{SA}$ ). (c) DA polymerization at $\mathrm{pH} 7.4$, in the dark or under UV, with $0 \mathrm{wt} \%, 0.4 \mathrm{wt} \%$ and $1 \mathrm{wt} \% \mathrm{SA}$ with respect to dopamine. (d) DA polymer ization at $\mathrm{pH} 9.5$, in the dark or under UV, with 0 wt\%, 0.4 wt\% and 1 wt\% SA with respect to dopamine. 
as a radical scavenger, which reacts with ROS formed under sunlight irradiation. Therefore it is possible to quickly consume SA by applying UV irradiation. Fig. 2 b shows the UVvis curves of different DA solutions ( $\mathrm{pH}$ 8.5) exposed to UV irradiation. As the figure illustrates, DA starts to oxidize and polymerize immediately after UV irradiation ( $c f$. Fig. S2f-h†) even in the presence of $1 \mathrm{wt} \%$ of SA in solution. The absorbance of all solutions increased significantly after irradiation for just 1 min (Fig. S2†).

In order to investigate the effect of $\mathrm{pH}$ on DA polymerization in the presence of SA, we performed the tests also at $\mathrm{pH}$ 7.4 and 9.5 (Fig. S3† and Fig. 2c, d), and the results indicated that SA exhibits a very similar inhibition effect at both pHs, although the DA polymerization in the dark at $\mathrm{pH} 7.4$ does not happen even without SA. At the same time, addition of SA did not change the $\mathrm{pH}$ of the solutions, indicating that the observed inhibition of DA polymerization by SA was not caused by reduction of $\mathrm{pH}$. Similar to the dopamine behavior at $\mathrm{pH} 8.5$, UV irradiation of the solutions at $\mathrm{pH} 7.4$ or 9.5 eliminated the inhibition effect and triggered the polymerization (Fig. 2c, d and Fig. $\mathrm{S} 3 \dagger$ ).

Another mechanistic question was whether the observed inhibition of DA polymerization in the presence of SA was due to possible oxygen consumption via oxidation of SA. The role of consumption of $\mathrm{O}_{2}$ by SA in buffer solutions at $\mathrm{pH} 8.5$ was investigated by monitoring the change in SA and $\mathrm{O}_{2}$ concentrations based on their respective oxidation and reduction currents in cyclic voltammetry. The results demonstrate that $\mathrm{O}_{2}$ is detected in the solutions even after 1 hour in high SA concentrations (Fig. S4a $\dagger$ ) and the SA concentration only decreases slightly after $9 \mathrm{~h}$ exposure to air (Fig. 3a). This demonstrates that the uncatalyzed reaction between $\mathrm{SA}$ and $\mathrm{O}_{2}$ is slow under basic conditions and, therefore, $\mathrm{O}_{2}$ removal from the solution caused by the reaction with SA is not the reason for inhibition of DA polymerization. Thus, this effect should be attributed to the reduction of DA quinone by SA that effectively counterbalances the rate of DA oxidation by $\mathrm{O}_{2}$ (Fig. 1).

In order to confirm the postulated inhibition effect of SA and the UV acceleration effect on dopamine polymerization at $\mathrm{pH}$ 8.5, we use electroanalytical methods to monitor the change in the SA oxidation peak response as a measure of the SA concentration (Fig. 3). It should be mentioned that we do not use the electro-oxidation of DA for quantitative purposes here because the expected small change in DA concentrations are hidden by fluctuations in peak currents due to the complex electrochemistry of DA involving proton-coupled electron transfer and due to the possible coupled homogeneous reactions both in the dark and under UV. While DA and SA can be easily detected by $\mathrm{CV}$ in separate solutions, their oxidations on a glassy carbon electrode take place within a narrow potential window and lead to signal overlap (Fig. $\mathrm{S} 4 \mathrm{~b} \dagger$ ). Discrimination of their respective signal in SA-DA mixtures is achieved by means of differential pulse voltammetry (DPV) with specifically activated glassy carbon electrodes. ${ }^{41}$ The SA oxidation in the SA-DA mixtures appears as a shoulder of the larger DA oxidation peak (Fig. 3d). The signals for SA in
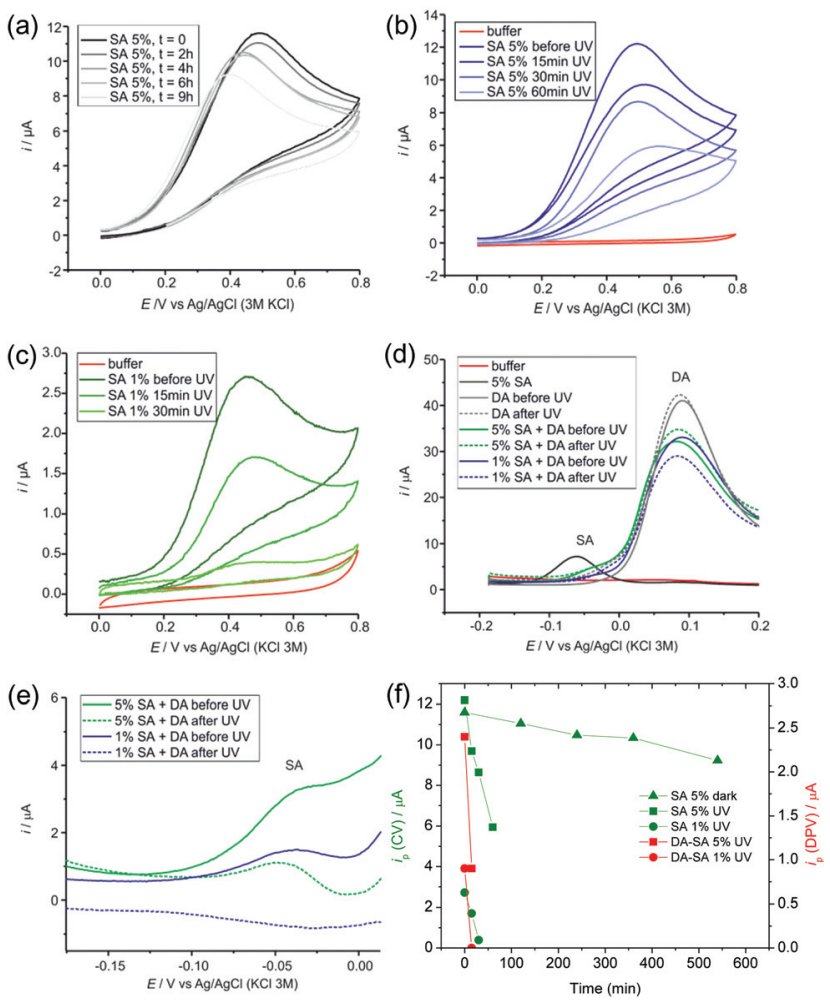

Fig. 3 Electro analytical investigation of the inhibition effect of SA and the UV acceleration effect on dopamine polymerization at $\mathrm{pH}$ 8.5. (a) Cyclic voltammogram (CV) in buffer with 5 wt\%. SA vs. time ( $t=0$, $2 \mathrm{~h}, 4 \mathrm{~h}, 6 \mathrm{~h}, 9 \mathrm{~h}$ : from black to light grey) in air in the dark. (b) CV in buffer with SA $5 \%$ vs. UV illumination time $(t=0,15 \mathrm{~min}, 30 \mathrm{~min}, 60 \mathrm{~min}$ : from dark blue to light blue). (c) CV in buffer with 1 wt\% SA vs. UV illumi nation time ( $t=0,15 \mathrm{~min}, 30 \mathrm{~min}$, from dark green to light green). (d) Differential pulse voltammetry (DPV) in Tris buffer (10 $\mathrm{mM} \mathrm{pH} \mathrm{8.5)}$ with $\mathrm{KCl}(0.1 \mathrm{M})$ at a scan rate of $100 \mathrm{mV} \mathrm{s}^{1}$ at activated glassy carbon disk electrodes: buffer (red), SA 5\% only (black), DA only (grey), SA 5\%+ DA (green) and SA 1\% + DA (blue) before (solid lines) and after (dashed lines) 15 min UV light illumination. (e) DPV of SA 5\% + DA (green) and SA $1 \%$ + DA (blue) before (solid lines) and after (dashed lines) 15 min UV light illumination after subtraction of the corresponding DPV of DA only. (f) Peak currents $\left(i_{p}\right)$ for SA oxidation $v s$. time for buffer with SA exposed to air in the dark ( $5 \mathrm{wt} \% \mathrm{SA}$ ) and under UV ( $1 \mathrm{wt} \%$ and $5 \mathrm{wt} \% \mathrm{SA}$ ) from CV (left $Y$ axis) and for buffer with SA and DA exposed to air under UV ( $1 \mathrm{wt} \%$ and $5 \mathrm{wt} \% \mathrm{SA}$ ) from DPV (right $Y$ axis). All the SA concentrations above are stated with respect to DA.

SA-DA mixtures are obtained by subtraction of the corresponding DPV of DA only solutions (Fig. 3e). Monitoring of SA under UV ( $\mathrm{pH}$ 8.5) by means of CV and DPV demonstrates that the oxidation rates of SA increased at least 20 fold (Fig. 3f, green symbols) in the absence of DA and by a further 5 -fold in its presence (Fig. 3f, red symbols). This phenomenon can be attributed to the formation of reactive oxygen species (ROS) from dissolved oxygen under UV irradiation, such as singlet oxygen $\left({ }^{1} \mathrm{O}_{2}\right)$, superoxide radicals $\left(\mathrm{O}_{2}^{-\cdot}\right)$, or hydroxyl radicals $\left({ }^{\circ} \mathrm{OH}\right)$, that have a twofold function: (a) rapid oxidative consumption of SA functioning as a reductant ${ }^{26,42,43}$ and (b) acceleration of the DA oxidation rate. ${ }^{26,29}$ The hypothesis was also confirmed by an experiment where the UV light was applied at 

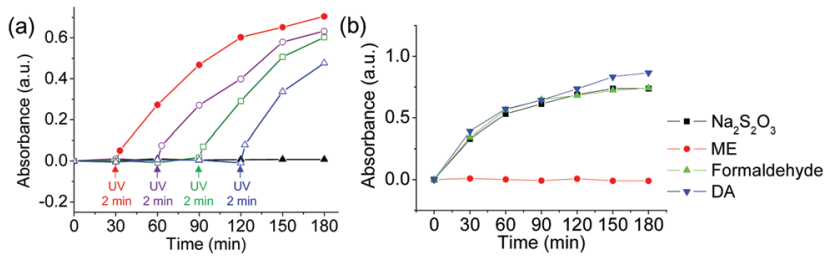

Fig. 4 UV triggering of dopamine polymerization and the inhibition effect of different reducing reagents. (a) Dopamine polymerization trig gered by short 2 min UV pulses performed at different time points ( $1 \mathrm{wt} \%$ of SA). No polymerization would be observed in $3 \mathrm{~h}$ without UV irradiation, however, after 2 min UV irradiation at any time points, the inhibition effect would be eliminated and dopamine starts to polymerize. (b) Effect of different reducing reagents, sodium thiosulfate, mercapto ethanol (ME) and formaldehyde on dopamine polymerization, compare with dopamine solution without an additional reducing reagent. The concentration of reducing reagents is $1 \mathrm{wt} \%$ with respect to dopamine.

different time points, thereby triggering DA polymerization "on demand" (Fig. 4a). Therefore, with the cooperation of SA and UV irradiation, by combining two natural roles of SA, control of DA polymerization in basic solutions was easily achieved.

To investigate the inhibition effect of different reducing reagents on dopamine polymerization, several commonly used reducing reagents, such as sodium thiosulfate, formaldehyde and mercaptoethanol (ME), were added to the dopamine solution $(\mathrm{pH}$ 8.5) and UV-vis spectra of the solutions were recorded. The results (Fig. 4b) showed that, only ME possessed a similar inhibition effect to SA, while for the solution containing sodium thiosulfate and formaldehyde, no inhibition effect was observed. The reason for this could be attributed to the oxidation-reduction potential of the reducing reagents. Dopamine is known as a reducing reagent (reported redox potential value: $403 \mathrm{mV} v s$. SHE at $\mathrm{pH} 7^{44}$ ), therefore in order to reduce dopamine quinone back to dopamine, the reducing reagent should have a lower redox potential than dopamine $\left(300 \mathrm{mV} v s\right.$. SHE for $\mathrm{SA}^{45}$ and $-260 \mathrm{mV} v s$. SHE for ME at $\mathrm{pH} 7^{46}$ ). The influence of a polyphenol, pyrogallol (PG), on dopamine polymerization was also tested (Fig. S5†).

Long-term control of DA polymerization under basic conditions could be achieved simply by increasing the SA concentration and the time of irradiation. As shown in Fig. 5a, the DA solution prepared at $\mathrm{pH} 8.5$ starts to polymerize immediately without SA. Adding $5 \mathrm{wt} \%$ (with respect to $2 \mathrm{mg} \mathrm{mL}^{-1} \mathrm{DA}$, i.e. $0.1 \mathrm{mg} \mathrm{mL}^{-1}$ ) of SA inhibits DA polymerization for over $24 \mathrm{~h}$ in the dark. Nevertheless, it is possible to initiate polymerization by a short 6 min pulse of UV irradiation (Fig. 5a). SA can also inhibit DA polymerization after its onset. As Fig. 5b illustrates, adding $5 \mathrm{wt} \%$ of SA to a polymerizing DA solution terminates the polymerization for at least $3 \mathrm{~h}$. Thus, by combining SA and UV irradiation, one can achieve even better control of DA polymerization in basic solutions. As shown in Fig. 5c, DA polymerization was completely inhibited by adding $1 \mathrm{wt} \%$ of SA (with respect to DA, $0.02 \mathrm{mg} \mathrm{mL}^{-1}$ ). However, UV irradiation of the solution for only $5 \mathrm{~min}$ an hour after adding
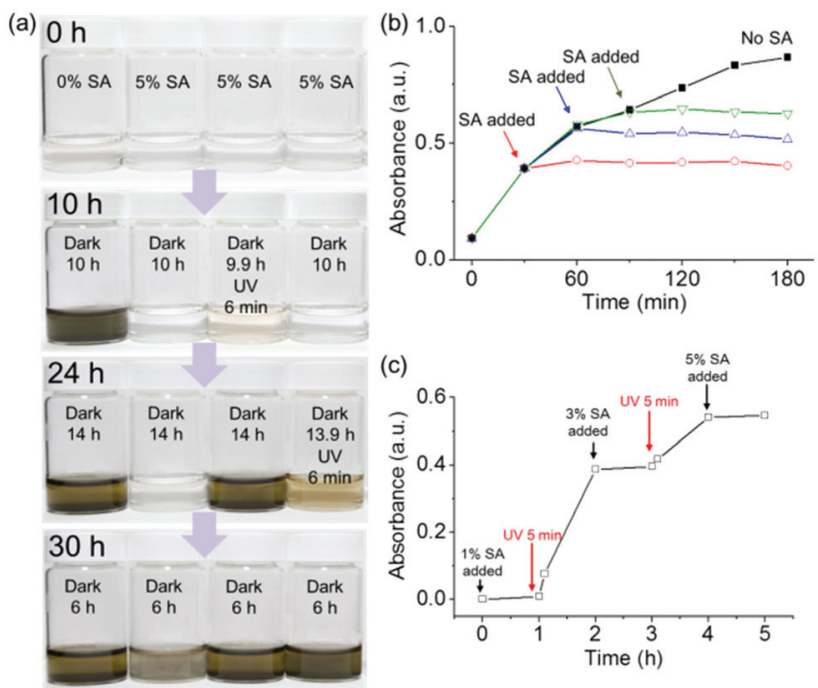

Fig. 5 Controlling DA polymerization under basic conditions. (a) Long term test for UV controlled DA polymerization. Adding $5 \mathrm{wt} \%$ of SA to a $2 \mathrm{mg} \mathrm{mL}{ }^{1}$ DA solution in Tris buffer ( $\mathrm{pH}$ 8.5) inhibits DA polymerization for more than $24 \mathrm{~h}$ (as opposed to immediate polymerization without SA). Polymerization can be initiated at any time point by a short $6 \mathrm{~min}$ UV pulse. (b) The graph illustrates the means of stopping incipient DA polymerization by adding $5 \mathrm{wt} \%$ of SA to a $2 \mathrm{mg} \mathrm{mL}{ }^{1} \mathrm{DA}$ solution ( $\mathrm{pH}$ 8.5, Tris buffer). (c) Controlling DA polymerization via the combined use of SA and UV light. $2 \mathrm{mg} \mathrm{mL}^{1}$ DA solution with $1 \mathrm{wt} \%$ of SA was placed in the dark for $1 \mathrm{~h}$, followed by 5 min UV irradiation to initiate polymeriz ation. 3 wt\% of SA was then added to the solution $1 \mathrm{~h}$ after the start of polymerization, resulting in its termination. This inhibition initiation cycle was repeated twice. Both graphs (b) and (c) display the solution's absorbance at $420 \mathrm{~nm}$. All the percentages of SA are given relative to the amount of DA in the solutions.

SA triggered DA polymerization in the dark, leading to a gradual increase in the solution's absorbance at $420 \mathrm{~nm}$. After adding $3 \mathrm{wt} \% \mathrm{SA}$ at the $2 \mathrm{~h}$ time point, DA polymerization stopped and the solution's absorbance remained constant for $1 \mathrm{~h}$. UV irradiation for 5 min then restarted the polymerization (Fig. 5c). This "inhibition-initiation" cycle was repeated several times by the sequential addition of SA and UV irradiation. However, it should be emphasized that as DA polymerization proceeds, the amount of SA required to inhibit polymerization also increased.

Further insights in the mechanism of the UV-SA assisted DA polymerization are gained from the comparison of experiments with a low and high SA concentration for a given UV irradiation intensity and duration. For SA concentrations that are low enough for its full consumption during UV illumination (SA 1 wt\% in DA under 15 min UV, Fig. S6a† and Fig. 6a), the polymerization is initiated during irradiation and carries on in the dark (Fig. 6a). In contrast, for SA concentrations that are sufficiently high to prevent its full consumption (e.g. SA $5 \mathrm{wt} \%$ with respect to DA under $15 \mathrm{~min} \mathrm{UV}$ ), polymerization is observed during illumination but is halted in the dark phase (Fig. S6b $\dagger$ and Fig. 6b). Eventually the SA is fully consumed by the slow reaction with $\mathrm{O}_{2}$ in the dark and polymerization 

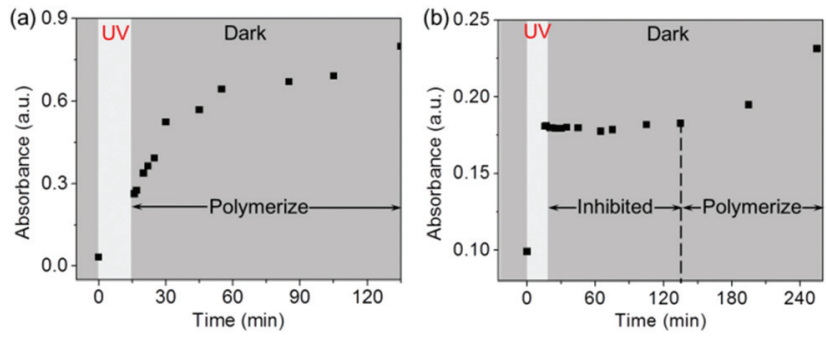

Fig. 6 Time dependent absorbance change of UV irradiated dopamine solutions at $\mathrm{pH} 8.5$, with (a) $1 \mathrm{wt} \%$ and (b) $5 \mathrm{wt} \%$ of SA (with respect to dopamine). After $15 \mathrm{~min}$ UV irradiation at $365 \mathrm{~nm}$, dopamine solution with $1 \mathrm{wt} \%$. SA starts to polymerize in the dark. Dopamine solution with 5 wt\% SA is still inhibited after irradiation, however, the absorbance changes of the solution before and after irradiation indicate that dopa mine polymerization occurred during the irradiation.

restarts after a lag time (Fig. 6b). The postulated mechanism for UV-SA assisted DA polymerization is shown in Fig. 1. SA is a reducing agent that reacts with oxygen in aqueous solutions, resulting in dehydroascorbic acid and hydrogen peroxide, ${ }^{47,48}$ which decomposes to form water and oxygen under basic conditions. ${ }^{49,50}$ In DA solutions without SA, DA reacts with oxygen to produce different quinones, followed by the polymerization step. However, SA inhibits polymerization of DA by fast reduction of quinones to catechols (Fig. 1). The DA polymerization under UV even before complete oxidation of SA (Fig. 1) demonstrates that the UV generated ROS accelerates the rates of DA oxidation to a greater extent than the rates of reduction of DA quinones by SA. Hence, a sufficient concentration of DA quinone is generated to enable its polymerization. When the UV illumination is sufficient for complete SA oxidation, then DA polymerization carries on unimpeded in the dark (Fig. 1).

Our method can be applied to the PD coating process to achieve controlled PD deposition. The coating process is almost the same as the normal procedure for PD coating, with a little SA added and a pulse of UV irradiation (Fig. 7a). Comparing PD surfaces formed by the normal process (coating for $24 \mathrm{~h}$ ) and the SA assisted process (inhibiting $6 \mathrm{~h}$, then UV $6 \mathrm{~min}$ and coating $24 \mathrm{~h}$ ), no significant difference is observable with the naked eye (Fig. 7b), SEM (Fig. 7c and d), or IRRAS (Fig. S7a $\dagger$ ). The thickness of both surfaces was $\sim 17 \mathrm{~nm}$ (by AFM test). The obtained surfaces were both secondary modified with dodecanethiol. The static WCA on both surfaces increased from $\sim 45^{\circ}$ to $\sim 100^{\circ}$ (Fig. S7b and $\mathrm{c} \dagger$ ), indicating that the PD deposited using the UV-SA method exhibits the same reactivity as the standard PD coatings formed without SA. ToF-SIMS analyses (negative secondary ion polarity) of the PD layer deposits with or without SA $(10 \mathrm{mM}$ Tris, $\mathrm{pH}$ 8.5) resulted in very similar spectra (Fig. S8 $\dagger$ ), indicating identical composition of both PD surfaces. No difference was observed at $m / z=175$, thus SA was not incorporated into the PD layer during the deposition. Moreover, dynamic SIMS analyses (with argon cluster bombardment) on the two PD layers showed very similar tendencies of the decrease/increase of $\mathrm{PD} /$ substrate

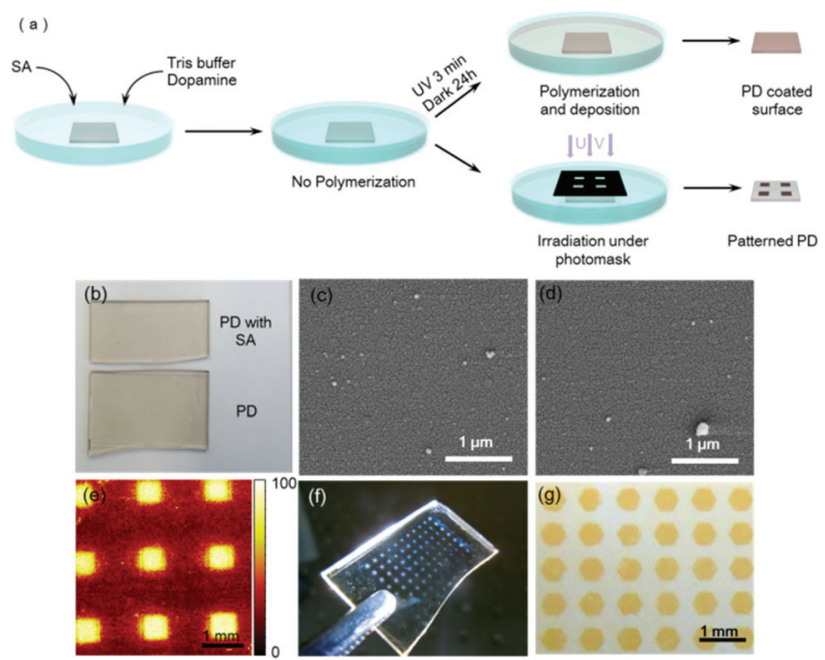

Fig. 7 Characterization of polydopamine coated substrates. (a) Illustration presenting the principle of UV SA assisted dopamine polymerization and PD deposition. (b) Photographs of glass slides coated with polydopamine obtained by using a standard base induced PD deposition (bottom sample) and via UV triggered dopamine polymerization in the presence of SA (UV SA) (top sample). The coatings were performed in Petri dishes without stirring. (c) SEM images of the PD coated glass; standard base induced dopamine polymerization, and (d) coated with PD using the UV SA controlled polymerization. (e) ToF SIMS CN intensity map of a glass surface patterned with PD using the UV SA method. (f) Image of a silver nanoparticle pattern on glass under white light (photo from the opposite side), obtained by immersing a PD patterned glass slide in $1 \mathrm{mg} \mathrm{mL}{ }^{1} \mathrm{AgNO}_{3}$ solution for $24 \mathrm{~h}$. Silver nanoparticles deposit on the PD coated areas, leading to a visible pattern due to strong light scattering. (g) PD was patterned on a porous polymer surface, by using the UV SA method with a photomask; after immersing for $24 \mathrm{~h}$ in $1 \mathrm{mg} \mathrm{mL}{ }^{1} \mathrm{AgNO}_{3}$ solution, a clear silver nanoparticle pattern could be observed.

ions during erosion (Fig. S9†), indicating an equal thickness of the two PD layers. Therefore it is concluded that the SA inhibition process does not affect the deposition of PD.

The deposition of PD on substrates is often performed under stirring to accelerate the process. We found that stirring did not affect the inhibition effect of SA and the obtained PD coating did not show any difference in the appearance or topography from the standard PD coating (Fig. S10 $†$ ).

We also compared the UV-SA method with our previously reported method, which controlled dopamine polymerization and PD deposition by UV irradiation of dopamine neutral or acidic solutions. ${ }^{26} 2 \mathrm{~h}$ UV irradiation was applied to $2 \mathrm{mg} \mathrm{mL}{ }^{-1}$ dopamine solutions at $\mathrm{pH} 8.5$ (1 wt\% SA with respect to dopamine) or $\mathrm{pH} 6.0$ (without additional additives), the morphology of the deposited PD surfaces (on glass substrate) was examined by AFM. The obtained images (Fig. S11†) indicate that the PD deposited at $\mathrm{pH} 8.5$ is smoother than that at $\mathrm{pH}$ 6.0. This might be attributed to the faster dopamine polymerization rate in basic solutions. ${ }^{26,51}$

Since SA efficiently inhibits DA polymerization in the dark and demonstrates almost no effect on DA polymerization under UV, PD patterns can be made using a photomask 
(Fig. 7a). This is demonstrated by the ToF-SIMS results (Fig. 7e and $\mathrm{S} 12 \dagger)$ and the formation of a silver nanoparticle pattern by PD assists electroless metallization on smooth (Fig. 7f) or porous surfaces (Fig. 7g).

\section{Conclusions}

In summary, inspired by the natural roles of vitamin $\mathrm{C}$ in the human body, we reported a new method for a light-triggered control of both the onset and termination of dopamine polymerization under basic conditions. We demonstrate that dopamine polymerization in basic solutions can be efficiently inhibited by adding a very small amount of sodium ascorbate (SA), that reduces reactive dopamine quinone. UV irradiation of this solution, however, leads to instantaneous dopamine polymerization, thereby making light-driven spatial and temporal control of dopamine polymerization under basic conditions possible. The mechanism of the light-driven SA-controlled dopamine (DA) polymerization has been studied using spectroscopic and electroanalytical methods. By this new method we can control dopamine polymerization without changing the common medium (base, oxidant) for polydopamine (PD) coatings, therefore the method is highly compatible with all existing applications for PD coatings. This new method allows the PD coating to be combined with many technologies which are not compatible with normal dopamine polymerization. We anticipate that the described method for the on-demand DA polymerization and PD deposition will lead to various applications and the development of novel functional coatings and patterns inspired by mussel adhesion.

\section{Experimental section}

Experimental details and supporting figures are provided in the ESI. $\dagger$

\section{Acknowledgements}

X. Du, J. S. Li and H. Zhang thank the China Scholarship Council for a Ph.D. scholarship. NP thanks the Cluster of Excellence RESOLV (EXC 1069) funded by the Deutsche Forschungsgemeinschaft for financial support. PL thanks the ERC Starting Grant (DropCellArray, ERC-2013-StG) and the Helmholtz Networking and Initiation Fund (grant VH-NG-621).

\section{Notes and references}

$1 \mathrm{H}$. Lee, S. M. Dellatore, W. M. Miller and P. B. Messersmith, Science, 2007, 318, 426-430.

2 Y. Liu, K. Ai and L. Lu, Chem. Rev., 2014, 114, 5057-5115.

3 M. E. Lynge, R. Van Der Westen, A. Postma and B. Städler, Nanoscale, 2011, 3, 4916-4928.

4 V. Ball, Biointerphases, 2014, 9, 030801.
5 Q. Ye, F. Zhou and W. Liu, Chem. Soc. Rev., 2011, 40, 42444258.

6 V. Ball, D. Frari, M. Michel, M. J. Buehler, V. Toniazzo, M. K. Singh, J. Gracio and D. Ruch, Bionanoscience, 2011, 2, 16-34.

7 N. Bandara, H. Zeng and J. Wu, J. Adhes. Sci. Technol., 2013, 27, 2139-2162.

8 B. P. Lee, P. B. Messersmith, J. N. Israelachvili and J. H. Waite, Annu. Rev. Mater. Res., 2011, 41, 99-132.

9 A. Napolitano, V. Ball, C. Chen and M. J. Buehler, Acc. Chem. Res., 2014, 47, 3541-3550.

10 D. R. Dreyer, D. J. Miller, B. D. Freeman, D. R. Paul and C. W. Bielawski, Chem. Sci., 2013, 4, 3796-3802.

11 T. S. Sileika, H. Do Kim, P. Maniak and P. B. Messersmith, ACS Appl. Mater. Interfaces, 2011, 3, 4602-4610.

12 M. Sureshkumar, D. Y. Siswanto and C. K. Lee, J. Mater. Chem., 2010, 20, 6948.

13 J. Kuang and P. B. Messersmith, Langmuir, 2012, 28, 72587266.

14 Q. Liu, B. Huang and A. Huang, J. Mater. Chem. A, 2013, 1, 11970-11974.

15 K. Yang, J. S. Lee, J. Kim, Y. Bin Lee, H. Shin, S. H. Um, J. B. Kim, K. I. Park, H. Lee and S. W. Cho, Biomaterials, 2012, 33, 6952-6964.

16 K. Sun, L. Song, Y. Xie, D. Liu, D. Wang, Z. Wang, W. Ma, J. Zhu and X. Jiang, Langmuir, 2011, 27, 5709-5712.

17 L. Zhang, J. Wu, Y. Wang, Y. Long, N. Zhao and J. Xu, J. Am. Chem. Soc., 2012, 134, 9879-9881.

18 K. Ni, H. Lu, C. Wang, K. C. L. Black, D. Wei, Y. Ren and P. B. Messersmith, Biotechnol. Bioeng., 2012, 109, 2970-2977.

19 H. Lee, B. P. Lee and P. B. Messersmith, Nature, 2007, 448, 338-341.

20 C. M. Preuss, T. Tischer, C. Rodriguez-Emmenegger, M. M. Zieger, M. Bruns, A. S. Goldmann and C. BarnerKowollik, J. Mater. Chem. B, 2014, 2, 36-40.

21 M. H. Ryou, J. Kim, I. Lee, S. Kim, Y. K. Jeong, S. Hong, J. H. Ryu, T.-S. Kim, J.-K. Park, H. Lee and J. W. Choi, Adv. Mater., 2013, 25, 1571-1576.

22 Y. Tian, Y. Cao, Y. Wang, W. Yang and J. Feng, Adv. Mater., 2013, 25, 2980-2983.

23 R. Li, K. Parvez, F. Hinkel, X. Feng and K. Müllen, Angew. Chem., Int. Ed., 2013, 52, 5535-5538.

24 J. Yan, L. Yang, M. F. Lin, J. Ma, X. Lu and P. S. Lee, Small, 2013, 9, 596-603.

25 K. Ai, Y. Liu, C. Ruan, L. Lu and G. M. Lu, Adv. Mater., 2013, 25, 998-1003.

26 X. Du, L. Li, J. Li, C. Yang, N. Frenkel, A. Welle, S. Heissler, A. Nefedov, M. Grunze and P. A. Levkin, Adv. Mater., 2014, 47, 8029-8033.

27 F. Ponzio, J. Barthès, J. Bour, M. Michel, P. Bertani, J. Hemmerlé, M. D’Ischia and V. Ball, Chem. Mater., 2016, 28, 4697-4705.

28 D. Perrot, C. Croutxé-Barghorn and X. Allonas, Polym. Chem., 2016, 7, 2635-2638.

29 C. Zhang, Y. Ou, W. Lei, L. Wan, J. Ji and Z. Xu, Angew. Chem., Int. Ed., 2016, 128, 3106-3109. 
30 V. Ball, J. Gracio, M. Vila, M. K. Singh, M.-H. MetzBoutigue, M. Michel, J. Bour, V. Toniazzo, D. Ruch and M. J. Buehler, Langmuir, 2013, 29, 12754-12761.

31 Q. Wei, F. Zhang, J. Li, B. Li and C. Zhao, Polym. Chem., 2010, 1, 1430.

32 M. Lee, S.-H. Lee, I.-K. Oh and H. Lee, Small, 2017, 13, 1600443.

33 I. You, H. Jeon, K. Lee, M. Do, Y. C. Seo, H. A. Lee and H. Lee, J. Ind. Eng. Chem., 2016, 46, 379-385.

34 J. a. Swift, Int. J. Cosmet. Sci., 2009, 31, 143-150.

35 K. Morisaki and S. Ozaki, Chem. Pharm. Bull., 1996, 44, 1647-1655.

36 Y. Ochiai, S. Kaburagi, K. Obayashi, N. Ujiie, S. Hashimoto, Y. Okano, H. Masaki, M. Ichihashi and H. Sakurai, J. Dermatol. Sci., 2006, 44, 37-44.

37 D. Darr, S. Combs, S. Dunston, T. Manning and S. Pinnell, Br. J. Dermatol., 1992, 127, 247-253.

38 M. Müller and B. Kessler, Langmuir: ACS J. Surf. Colloids, 2011, 27, 12499-12505.

39 J. Liebscher, R. Mrówczyński, H. a. Scheidt, C. Filip, N. D. Hădade, R. Turcu, A. Bende and S. Beck, Langmuir, 2013, 29, 10539-10548.

40 N. F. Della Vecchia, R. Avolio, M. Alfè, M. E. Errico, A. Napolitano and M. d'Ischia, Adv. Funct. Mater., 2013, 23, 1331-1340.
41 S. Thiagarajan, T. Tsai and S. Chen, Biosens. Bioelectron., 2009, 24, 2712-2715.

42 E. Hideg, C. Barta, T. Kálai, I. Vass, K. Hideg and K. Asada, Plant Cell Physiol., 2002, 43, 11541164.

43 D. Luo, X. W. Sun, H. T. Dai, Y. J. Liu, H. Z. Yang and W. Ji, Appl. Phys. Lett., 2009, 95, 151115.

44 U. Chandra, B. E. K. Swamy, O. Gilbert, S. S. Shankar, K. R. Mahanthesha and B. S. Sherigara, Int. J. Electrochem. Sci., 2010, 5, 1-9.

45 S. Steenken and P. Neta, J. Org. Chem., 1982, 86, 36613667.

46 G. M. Whitesides and W. J. Lees, J. Org. Chem., 1993, 58, 642-647.

47 M. P. Bradshaw, P. D. Prenzler and G. R. Scollary, Electroanalysis, 2002, 14, 546-550.

48 Z. Peng, B. Duncan, K. F. Pocock and M. a. Sefton, Aust. J. Grape Wine Res., 1998, 4, 127-135.

49 O. Špalek, J. Balej and I. Paseka, J. Chem. Soc., Faraday Trans., 1982, 78, 2349.

50 A. F. Smith and W. D. Nicoll, Ind. Eng. Chem., 1955, 47, 2548-2554.

51 H. W. Kim, B. D. McCloskey, T. H. Choi, C. Lee, M.-J. Kim, B. D. Freeman and H. B. Park, ACS Appl. Mater. Interfaces, 2013, 5, 233-238. 


\section{Repository KITopen}

Dies ist ein Postprint/begutachtetes Manuskript.

Empfohlene Zitierung:

Du, X.; Li, L.; Behboodi-Sadabad, F.; Welle, A.; Li, J.; Heissler, S.; Zhang, H.; Plumeré, N.; Levkin, P. A.

Bio-inspired strategy for controlled dopamine polymerization in basic solutions.

2017. Polymer chemistry

doi: $10.554 / I R / 1000068824$

Zitierung der Originalveröffentlichung:

Du, X.; Li, L.; Behboodi-Sadabad, F.; Welle, A.; Li, J.; Heissler, S.; Zhang, H.; Plumeré, N.; Levkin, P. A.

Bio-inspired strategy for controlled dopamine polymerization in basic solutions.

2017. Polymer chemistry, 8 (14), 2145-2151. doi:10.1039/C7PY00051K 